\section{Wenn zwei und zwei fünf ist}

\author{
E. Taverna
}

Wenn vom Generalisten die Rede ist, wird gerne behauptet, dass seine Dienstleistung mehr sei als die Summe aller gelernten Einzelfächer. Glaubt man dem Editorial von Ars Medici, dann finden sich die Grundversorger auf dem Abstellgleis. Der Allgemeinmedizin bleibe «der Charme des einfachen Daseins in der medizinischen Provinz", abgeschmettert vom universitären Establishment, wo sie am Rande ihr geduldetes Dasein fristet. Zu Recht wird darauf hingewiesen, dass «ihr bis heute nicht wirklich gelungen ist, sich in ihrer Eigenständigkeit zu beschreiben." Will sie nicht zur "Berufslehre» verkommen, muss sie zum lehrbaren, wissenschaftlichen Partner mutieren. Damit ist der alte Streit wieder neu aufgelegt, was den Generalisten vom Spezialisten unterscheide. Ja, was denn?

\section{Die Vorstellungen}

Verwirrend ist bereits die Definition dieses Generalisten. Im gleichen Heft ist die Innere Medizin der Inbegriff eines generalistischen Faches, dem allerdings die Zerstörung droht, weil die Subspezialisten selbständig sein wollen, oder sich gar, wie in Bern, mit den Chirurgen zu "Organdepartementen» zusammenschliessen. Damit sei der Nachwuchs für Klinik und Praxis in Gefahr. Was tut denn dieser HausarztInternist? Laut gleichem Artikel behandelt er vor allem ältere, polymorbide Patienten, von denen er etwa einen Zehntel an Spezialisten weiterweist. Seine Führungsfunktion besteht aus Koordinieren, Prioritäten setzen und Ressourcen verteilen.

Im gleichzeitig erschienenen Primary-Care-Heft sucht ein britischer Kollege nach einer Antwort auf die Frage "Being a general practitioner: what it means." McWhinney zieht eine Bilanz seiner langen Praxisjahre, wobei er die langdauernde Patientenbeziehung als etwas Besonderes herausstreicht. "Cumulative knowledge in a long term relationship gives us great advantages. It means that every new event can be understood in the context of a life story." Er plädiert für ein "organismisches" Konzept, das Körper und Geist oder klinische und existentielle Medizin vereinigt. Der Allgemeinarzt soll holistischkomplementär denken und engagiert, mitfühlend, emotional intelligent, ausgeglichen und aus Selbsterkenntnis handeln. Letzteres kann schmerzhaft sein und braucht einen Mentor als guten Lehrer. Seine Weisheit gründet im aufmerksamen Zuhören, eine Kunst, die ihn zu einem anderen Arzt macht, weil sie ihn persönlich verändert.
Ian R. McWhinney hat zusammen mit Hannes G. Pauli und Kerr L. White eine Arbeit publiziert mit dem Titel: «Medizinische Ausbildung, Forschung und wissenschaftliches Denken im 21. Jahrhundert." Die Autoren definieren eine neue Ausbildung für Studenten auf der Basis einer umfassenden Kritik biomedizinischer Denkmodelle. Sie fordern und begründen eine wissenschaftliche Neuorientierung, ein «biosemiotisches» Denken, das dem Vorherrschen des mathematisch-materialistischen Paradigmas eine Ende bereiten soll. Die Trennung von Natur- und Geisteswissenschaften wird mit Hinweisen auf die Erkenntnisse des Medizinsoziologen Antonovsky und des Psychosomatikers Uexküll aufgehoben. Sie formulieren eine vorläufige Liste möglicher Forschungsinhalte für eine empirische Forschung für Allgemeinärzte und andere Generalisten. Die medizinische Ausbildung soll «den moralischen Aspekt mit einbeziehen durch das $\mathrm{Zu}$ lassen und Fördern von echten Gefühlen, Reflexion, Fantasie, Neugier und die Fähigkeit zur Überprüfung des Selbst, einschliesslich der Korrektur falscher Gefühle.» Gefordert wird auch eine Neuverteilung von politischer und professioneller Macht auf «der Ebene von Familie, Gemeinde, Region, nationalen und internationalen Gremien und Behörden". Berufsangehörige der Grundversorgung im Kontakt mit den am wenigsten festgefahrenen Anteilen akademischer Hierarchien sollen die Führung einer überfälligen Entwicklung übernehmen. Die Debatte um eine neue Medizin wurde in der Weltwoche vom 17. Mai 2001 unter dem Titel "Mediziner statt Maschinen" auf der Grundlage eines Gesprächs mit Hannes Pauli öffentlich gemacht.

\section{Die Verhältnisse}

Viele lobenswerte Eigenschaften werden erwähnt, die auch jedem anderen Zeitgenossen gut anstünden. Der Kunde sucht aber in erster Linie einen guten Handwerker und nicht einen Freund, Priester oder Philosophen. Die praktischen Fähigkeiten des Allgemeinmediziners oder Familienarztes sind selten mehr ein Thema. Der kollegiale Frieden ist eine brüchige Sache. Das weiss auch das "Kollegium für Hausarztmedizin» und verteilt die Grundversorgung neutral auf vorhandenen Anbieter. Damit werden zwar lästige Auseinandersetzungen vermieden, Reformen leider auch. Was müsste der Generalist ausser Moral und Organisationstalent noch bieten können? Die kompetente medizinische, chirurgische, gynäkologische und pädiatrische Erledigung von Alltagsproblemen, also des weitaus grössten Teils der ambulanten Nachfrage. Doch die Verhältnisse sind nicht so, weil die niedergelassenen Organspezialisten auf die gleichen Einnahmequellen angewiesen sind. Das verteuert zwar die Versorgung, aber das "doctor shopping" lässt sich ein grosser Teil der Bevölkerung, auch durch steigende Krankenkassenprämien, nicht nehmen. Die Zweiklassenmedizin ist eine Tatsache, gefördert durch die Dauerberieselung mit Sprechstunden- 
sendungen, die zudem falsche Prioritäten setzen. Das gewünschte, emotional-spirituell-ganzheitliche gewisse Etwas wird inzwischen mit staatlicher Hilfe in das Segment der alternativ-komplementären Anbieter entsorgt.

Das Bedürfnis nach einer individuellen Totalbetreuung kann die Medizin nicht stillen, es ist auch nicht ihre Aufgabe. Wer sich in narzisstischer Weise unablässig mit dem eigenen Wohl beschäftigt, ignoriert die wachsenden vitalen Bedürfnisse einer verarmten Mehrheit, die wir direkt oder indirekt ausbeuten.
Keine der zitierten Arbeiten geht auf spezifische Frauenfragen ein, obwohl der Nachwuchs mehrheitlich weiblich sein wird. Auch das Verdienen wird ausgeblendet. Dabei möchten alle lieber mehr Geld als weniger. Selbsterkenntnis und Weisheit haben keinen Marktwert. Die guten Ausbildungsvorsätze landen früher oder später im Shredder der Tarifsysteme. Das Dauergeklingel der Ladenkasse macht taub. Konform der kapitalistischen Gesellschaft sind Klinik und Praxis umsatzorientiert. Wehe, wenn sie es nicht sind. An dieser Realität kommt kein beschworener Paradigmawechsel vorbei.

\section{Ein totgeschwiegenes Buch. Ist es auferstanden?}

\author{
H. G. Pauli
}

Studierenden) sei noch über erfolgreiche Errungenschaften der Medizin berichte oder von Sponsoren gefördert werde. So wurde der Autor, dem seine Botschaft ein Anliegen war, mit DM 10000 sein eigener Sponsor.

Gerald Ulrich geht vom Begriff und vom Status quo der biologischen Psychiatrie aus. Er sieht die Realität dieses Fachbereiches als Produkt «eines immer weiteren Auseinanderdriftens vom biologischen und psychosozialen Ansatz». So würden z.B. als Aufnahmebedingung in die Deutsche Gesellschaft für Biologische Psychiatrie lediglich Interesse oder wissenschaftliches Arbeiten auf dem Gebiet von Neuropharmakologie und Pharmakopsychiatrie gefordert. "Wäre es da nicht viel erhellender und zutreffender, anstatt von sbiologisch von (somatologisch oder noch präziser - von ıhysikochemisch zu sprechen?» Damit ist gleichzeitig die Problematik des Biologiebegriffes für die gesamte Medizin, ja der gesamten Lebenswissenschaften aufgezeigt: "Unter Biologie versteht man heute eine sogenannte Grundlagenoder Laborwissenschaft, die das Phänomen Leben ausklammert."

In dichter und konziser Weise belegt der Autor die Stichhaltigkeit dieser provokativen Aussage. Aus dieser brillanten Analyse können hier nur einige wenige Themen herausgegriffen werden, die allesamt mit den vieldiskutierten konzeptionellen, gesundheitspolitischen, institutionellen, praktischen und ökonomischen Problemen der heutigen Medizin in Verbindung stehen.

Es ist von der "wachsenden Kluft zwischen der Grundlagenwissenschaft und der Praxis" die Rede, einer Grundlagenwissenschaft, die in unseren etablierten Institutionen im wesentlichen auf die materielle und damit auf die technische Ebene beschränkt ist.

"Spitzenleistungen" der Medizin werden dann auf ebendiesen Ebenen gesehen. Der entsprechend leistungserbringende Arzt ist demzufolge ein Gesundheitsingenieur.
Korrespondenz:

Prof. Dr. med. Hannes G. Pauli

Oranienburgstrasse 13

CH-3013 Bern 
«Die ärztliche Diagnose gewann dadurch den Status eines naturwissenschaftlichen Erkennens.» Die diagnostischen Kategorien, die daraus hervorgehen, erscheinen dann als "von Patienten unabhängige Einheiten». Es kommt damit zum "Aufstellen von Taxonomien aller nur denkbaren Naturerscheinungen [...] über die medizinischen Nosologien bis hin zur Kartographierung des gesamten menschlichen Genoms», womit ein "unidirektional-lineares Krankheitskonzept" angestrebt wird.

Zum so skizzierten medizinischen Denken stellt sich die Frage nach einer alternativen Sicht (nicht im Sinne der etablierten "Alternativmedizin») unter der Voraussetzung, dass der Begriff «Bio-» nun effektiv "Leben» in seiner ganzen heute zugänglichen Phänomenologie umfasst. Lebende Systeme erweisen sich dann als "nichttriviale selbstreferentielle Maschinen». Selbstreferentialität meint, dass sich das System in einem selbstdeterminierten Zustand befindet und dass dadurch der Effekt jeder Ausseneinwirkung vom aktuell gegebenen Systemzustand abhängt. Triviale (technische) Maschinen sind demgegenüber stets aussendeterminiert, sei es auf linear-kausale oder auf zirkulär-kausale Weise.

Als Konsequenz einer derartig erweiterten Sicht des Lebens- bzw. Humanbereiches wird eine Überwindung des "fatalen Schismas von Biologie und
Geisteswissenschaft» gefordert, das heute als «Fortschrittshindernis ersten Ranges» gesehen werden muss. Die Integration von Natur- und Geisteswissenschaften ist gleichzeitig Voraussetzung für eine "salutogenetische" Forschung, die einen "beschreibenden" gegenüber dem messenden, "erklärenden" Ansatz der etablierten pathogenetischen Forschung durchzusetzen hat. Auch im Bereich der Methodik muss nach innovativen Lösungen gesucht werden. So lässt sich beispielsweise der Individualität lebender Systeme biometrisch nur durch sequentielle Einzelanalysen gerecht werden. Beim primär-gruppenstatistischen Design wird die Individualität des einzelnen Elementes der Gruppe preisgegeben.

Mit dieser partiellen Aufzählung einiger inhaltlicher Schwerpunkte und deren Konsequenzen aus Ulrichs Analyse wird vielleicht verständlich, dass eine über Jahrhunderte gewachsene und konsolidierte Profession es vorzieht wegzusehen und die Diskussion $\mathrm{zu}$ verweigern.

Darf es beim Erröten bleiben?

\section{Literatur}

1 Ulrich G. Biomedizin - Die folgenschweren Wandlungen des Biologiebegriffes. Stuttgart, New York: Schattauer; 1997. 203 Seiten. ISBN 3-7945-1817-99. 